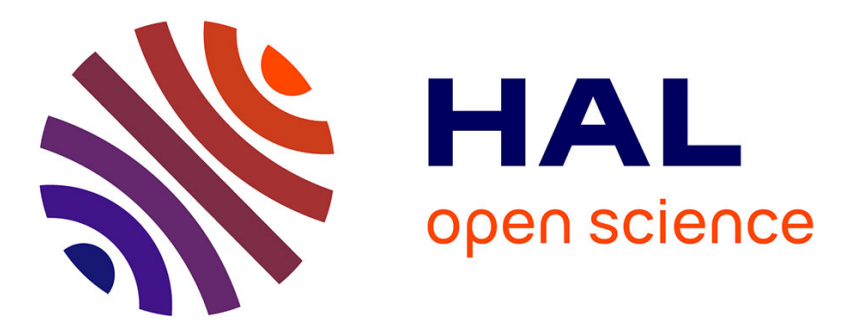

\title{
Towards real-time assessment of anisotropic plate properties using elastic guided waves
}

Nicolas Bochud, Jérôme Laurent, Francois Bruno, Daniel Royer, Claire Prada

\section{To cite this version:}

Nicolas Bochud, Jérôme Laurent, Francois Bruno, Daniel Royer, Claire Prada. Towards real-time assessment of anisotropic plate properties using elastic guided waves. Journal of the Acoustical Society of America, 2018, 143. hal-02335140

\section{HAL Id: hal-02335140 \\ https://hal.science/hal-02335140}

Submitted on 28 Oct 2019

HAL is a multi-disciplinary open access archive for the deposit and dissemination of scientific research documents, whether they are published or not. The documents may come from teaching and research institutions in France or abroad, or from public or private research centers.
L'archive ouverte pluridisciplinaire HAL, est destinée au dépôt et à la diffusion de documents scientifiques de niveau recherche, publiés ou non, émanant des établissements d'enseignement et de recherche français ou étrangers, des laboratoires publics ou privés. 


\section{Towards real-time assessment of anisotropic plate properties using elastic guided waves}

Nicolas Bochud, Jérôme Laurent, François Bruno, Daniel Royer, and Claire Prada

Citation: The Journal of the Acoustical Society of America 143, 1138 (2018); doi: 10.1121/1.5024353

View online: https://doi.org/10.1121/1.5024353

View Table of Contents: http://asa.scitation.org/toc/jas/143/2

Published by the Acoustical Society of America

\section{Articles you may be interested in}

Multichannel Multiple Signal Classification for dispersion curves extraction of ultrasonic guided waves

The Journal of the Acoustical Society of America 143, EL87 (2018); 10.1121/1.5022699

Multiple scattering and scattering cross sections

The Journal of the Acoustical Society of America 143, 995 (2018); 10.1121/1.5024361

Plane nonlinear shear waves in relaxing media

The Journal of the Acoustical Society of America 143, 1035 (2018); 10.1121/1.5023394

Deep convolutional neural networks for estimating porous material parameters with ultrasound tomography The Journal of the Acoustical Society of America 143, 1148 (2018); 10.1121/1.5024341

Dispersion curves for Lamb wave propagation in prestressed plates using a semi-analytical finite element analysis

The Journal of the Acoustical Society of America 143, 829 (2018); 10.1121/1.5023335

Modeling analysis of ultrasonic attenuation and angular scattering measurements of suspended particles The Journal of the Acoustical Society of America 143, 1049 (2018); 10.1121/1.5024233 


\title{
Towards real-time assessment of anisotropic plate properties using elastic guided waves
}

\author{
Nicolas Bochud, Jérôme Laurent, François Bruno, Daniel Royer, and Claire Prada ${ }^{a)}$ \\ Institut Langevin, ESPCI Paris, CNRS (UMR 7587), PSL Research University, 75005 Paris, France
}

\begin{abstract}
(Received 5 December 2017; revised 29 January 2018; accepted 29 January 2018; published online 22 February 2018)

A method to recover the elastic properties, thickness, or orientation of the principal symmetry axes of anisotropic plates is presented. This method relies on the measurements of multimode guided waves, which are launched and detected in arbitrary directions along the plate using a multielement linear transducer array driven by a programmable electronic device. A model-based inverse problem solution is proposed to optimally recover the properties of interest. The main contribution consists in defining an objective function built from the dispersion equation, which allows accounting for higher-order modes without the need to pair each experimental data point to a specific guided mode. This avoids the numerical calculation of the dispersion curves and errors in the mode identification. Compared to standard root-finding algorithms, the computational gain of the procedure is estimated to be on the order of 200. The objective function is optimized using genetic algorithms, which allow identifying from a single out-of-symmetry axis measurement the full set of anisotropic elastic coefficients and either the plate thickness or the propagation direction. The efficiency of the method is demonstrated using data measured on materials with different symmetry classes. Excellent agreement is found between the reported estimates and reference values from the literature. (C) 2018 Acoustical Society of America. https://doi.org/10.1121/1.5024353
\end{abstract}

[ANN]

Pages: $1138-1147$

\section{INTRODUCTION}

Elastic waves are frequently applied for nondestructive material characterization and have been used with success in the past decades. In particular, several attempts to identify the anisotropic stiffness tensor have been made, which is an essential task for modeling and evaluating the mechanical behavior of polycrystalline metals and composite materials. Initially, the anisotropic elastic properties of a number of materials have been determined through bulk waves measurements. ${ }^{1,2}$ Nonetheless, these experiments require the measurements to be performed along many propagation directions. In addition, for wave speed data processing, the correct identification of the two transverse waves is not trivial and some additional information is usually required for this purpose. ${ }^{3}$ Alternatively, there are several advantages in using elastic guided waves (EGWs) for the nondestructive evaluation of anisotropic materials. ${ }^{4}$ Their dispersive and multimodal nature is especially useful when it is desirable to use wavelengths larger than the plate thickness or when it is necessary to measure in-plane elastic properties, ${ }^{5}$ as the components of the elastic tensor affect each mode differently and with different sensitivities. ${ }^{6}$

To achieve the identification of anisotropic material properties, two issues have to be addressed. The first one is related to the ultrasonic measurement configuration and signal acquisition method, i.e., the use of both narrowband and broadband excitations has been reported. ${ }^{7}$ Second, a robust model-based inverse procedure is required to extract the elastic properties from the measured data. ${ }^{8}$ Narrowband

a)Electronic mail: claire.prada-julia@espci.fr signals are used to excite several pure modes by sweeping the frequency and varying the transducer angle of incidence, thus allowing the recording of phase velocities as discrete points over a broad frequency range by means of the phase shift method. ${ }^{5}$ This approach can be implemented in many ways using, for example, variable angle wedges in pitchcatch configuration, ${ }^{5}$ air-coupled transducers, ${ }^{9,10}$ oblique insonification of a test specimen that is fully immersed in water [so-called leaky Lamb wave (LLW) technique], ${ }^{6,11,12}$ or a piezoelectric transducer for the generation and full-field interferometric techniques for the signal detection. ${ }^{7}$ However, the aforementioned techniques require a cumbersome and non-portable equipment to control probe positions or angles of incidence.

In contrast, broadband signals contain more information than narrowband signals, but the separation of each mode contribution and the dispersive effects within each mode are key issues to address and require data processing. Broadband signals can be acquired with a diversity of techniques, like a piezoceramic transducer for emitting and a laser scanning Doppler vibrometer for sensing, ${ }^{13}$ laser-ultrasound, ${ }^{14,15}$ or line-focus acoustic microscopy. ${ }^{16}$ Spatio-temporal broadband signals recorded at equally spaced points are twodimensionally (2-D) Fourier transformed to obtain the frequency spectrum of the different propagating modes in the frequency-wave number plane. ${ }^{17}$ Then, for comparison with theoretical results, this information is generally processed to extract the ridges of the measured dispersion curves in terms of frequency-wave number data pairs. Although scarcely discussed in the literature, their automatic detection can be problematic due to mode superposition and limited spatial resolution. ${ }^{18}$ To carefully extract the dispersion curves, 
visual operator selection, ${ }^{19}$ peak-finding algorithms and interpolation, ${ }^{13}$ image tracing algorithm, ${ }^{16}$ matrix pencil method, ${ }^{20}$ and more advanced data processing algorithms, as reviewed in Refs. 21 and 22, have been developed, often at the cost of time-consuming procedures.

As a first limitation, most of the former techniques are designed for the analysis of signals captured in a pointwise scheme, and the change of the recording location is usually performed by mechanically displacing the receiver. Furthermore, measurements are generally carried out in a propagation plane that coincides with a principal symmetry plane of the material and the principal symmetry axes and plate thickness are assumed to be known. Last, to retrieve elastic properties, least-squares fitting criteria are frequently applied, i.e., a procedure is followed in order to minimize the sum of the squared differences between extracted experimental data and theoretical dispersion curves. For this approach, it is necessary to identify the branches of the dispersion curves prior to the inversion process. ${ }^{5,23}$ However, it should be noted that a clear identification of the modes in experimental data is uncertain, especially for higher-order modes. ${ }^{16,19}$ In this way, Karim et al. ${ }^{6}$ presented a numerical procedure for the inversion of LLW data to determine certain material properties of a composite laminate using a modified version of the simplex algorithm. For this approach to be effective, four broadband transducers were used to record the phase velocities over a wide frequency range and three measurements in different propagation directions were needed to recover the full set of independent elastic coefficients. In another related study, Yan et al. ${ }^{16}$ described a method for the inversion of the elastic properties of plates by line-focus acoustic microscopy using a hybrid particle swarm-based-simulated annealing optimization. However, this approach required tedious data processing for converting time-domain waveforms into dispersion curves and was solely applied to a thin isotropic plate. An alternative userindependent inversion scheme is thus highly desirable towards real-time applications.

To face these limitations, the primary objective of the present work is to develop an effective method to identify the geometric and anisotropic elastic properties of plate materials. To this end, an accurate and simple technique to measure multimode dispersion curves is proposed. This technique takes advantage of the advent of multi-element probes, together with multichannel electronics, initially devoted to medical imaging. Therefore, applications to material characterization are expanding, e.g., for cortical bone assessment $^{24,25}$ and structural health monitoring. ${ }^{26}$ This approach offers several advantages: (1) the acquisition procedure is significantly simpler than that delivered by other setups, such as the LLW technique and laser-based devices; (2) it requires minimal data processing and no extraction of the experimental data; and (3) it is well suited for field use (i.e., single-side access, no mechanical displacement). Although the probe induces leakage, we observe that it does not significantly modify the phase velocity of the propagating modes. ${ }^{27}$ In addition to the experimental technique, a decisive task is to develop a robust inverse procedure. To avoid the difficult pairing between experimental data points and theoretical guided modes, an objective function is built from the dispersion equation and directly evaluated on experimental data points. This objective function does not require the numerical calculation of the theoretical guided modes, and thus drastically reduces the computational costs for solving the inverse problem. Besides, we show that the method can also be applied to simultaneously recover the elastic properties and the propagation direction angle. Genetic algorithms are chosen for optimizing the objective function, owing to their flexibility in solving multiparametric inversion problem. ${ }^{28}$

The reconstruction method is applied to experimental data measured on materials with various symmetry classes. Results are presented for isotropic duralumin and fused quartz plates, a silicon wafer with cubic symmetry and a transversely isotropic titanium plate. The proposed inversion procedure is proven feasible to recover the full set of elastic coefficients, together with the plate thickness or the propagation plane, from a single dispersion curves measurement. The paper is structured as follows: the experimental setup used to measure guided waves is described in Sec. II. This section also presents the forward model used to predict the theoretical dispersion curves, along with the inverse procedure employed to retrieve the material properties. The results obtained for two case studies, i.e., the inference of plate properties for a known propagation direction and the inference of elastic properties and propagation angle for a known plate thickness are exposed in Sec. III. The results are compared to reference values and discussed in the light of computational costs, inverse problem errors, and measurement uncertainties in Sec. IV.

\section{MATERIALS AND METHODS}

Measurements of EGWs, along with appropriate modeling, have the potential for yielding estimates of waveguide properties such as thickness, elasticity and the angle of propagation with respect to the principal symmetry axes. Such a model-based approach requires solving a multiparametric inverse problem to match the experimental dispersion curves with the predicted guided modes. The experimental setup used to measure guided waves in anisotropic plates is first described. Then, the inverse procedure used to recover material properties is presented.

\section{A. Guided waves measurements and investigated samples}

EGWs measurements were performed using a commercially available $10-\mathrm{MHz}$ standard probe (Imasonic, Besançon, France), which consists in a 128-element linear transducer array. The array pitch was $0.25 \mathrm{~mm}$ and the dimensions of each rectangular element were $0.2 \times 10 \mathrm{~mm}^{2}$. The probe was mounted on a rotational stage (ESP 301 Motion Controller, Newport, CA), allowing measurements in any propagation direction $\phi$ with respect to the principal symmetry axes of the material. The probe was placed directly in contact with the sample using tap water for coupling. A Lecoeur Electronics Ltd system was used to transmit a broadband chirp signal at a $8-\mathrm{MHz}$ central frequency 
(a) Input signal

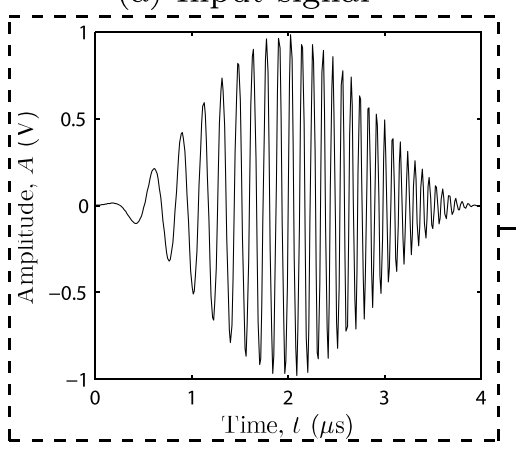

(b) Measurement configuration

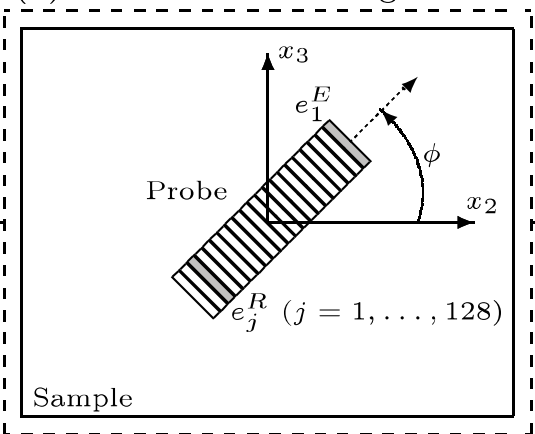

(e) Dispersion curves after thresholding

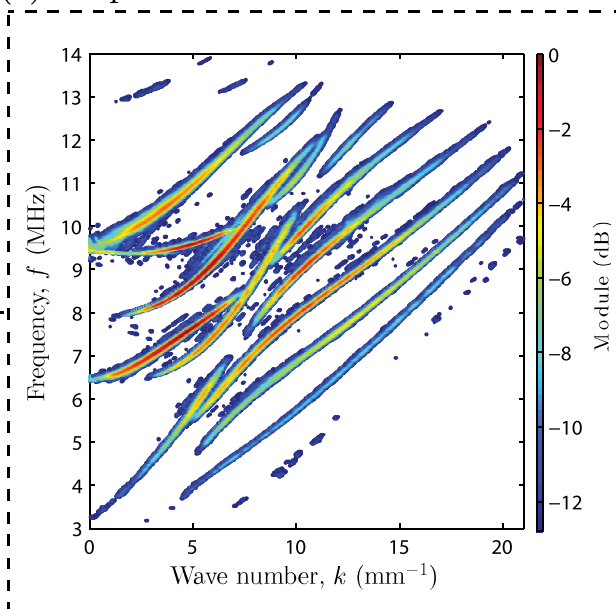

(c) Radio-frequency signals

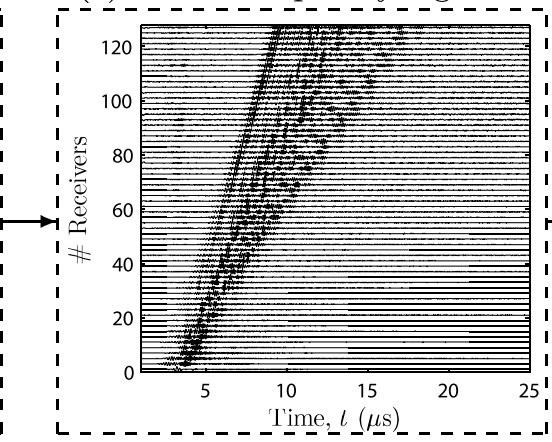

(d) Dispersion curves

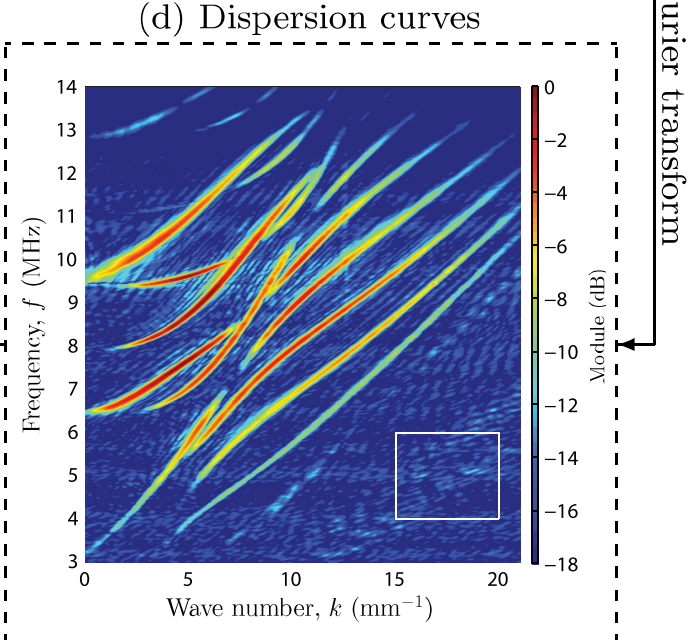

FIG. 1. (Color online) Flowchart of the acquisition and data processing: (a) broadband chirp signal used as excitation; (b) schematic view of the multi-element probe; (c) radio-frequency signals; (d) dispersion curves after applying a 2-D Fourier transform; (e) dispersion curves after denoising (i.e., the threshold was set according to the noise level evaluated within the area inside the white box); and (f) final experimental data.

( $-6 \mathrm{~dB}$ power spectrum spanning the frequency range from 4 to $12 \mathrm{MHz}$ ) and $4 \mu$ s duration to the first element $e_{1}^{E}$ and to record the received signals on all elements $e_{j}^{R}$ $(j=1, \ldots, 128)$ during $25 \mu \mathrm{s}$. For each propagation direction $\phi$, a set of 128 radio-frequency (RF) signals was digitized (12 bits, $80 \mathrm{MHz}, 8000$ samples).

The experimental dispersion curves of the guided modes were obtained as follows: (1) for each propagation direction $\phi$, the 128 RF signals were Fourier transformed with respect to time and space, ${ }^{17}$ and stored in a response matrix; (2) the mean noise level (in $\mathrm{dB}$ ) was evaluated in an area away from the guided modes (i.e., low phase velocity area), and all components of the response matrix below this threshold were set to zero; (3) the remaining $N$ non-zero components were stored into a $N \times 3$ matrix $\left[k_{n} f_{n} w_{n}\right](n=1, \ldots, N)$, where $k, f$, and $w$ stand for wave number, frequency and Fourier coefficients, respectively. It is worth mentioning that these data processing steps were only applied to remove a large part of the background noise and decrease the amount of data, but the dispersion curves still contain many spurious data (e.g., noise, side lobes). A flowchart of the acquisition and data processing is depicted in Fig. 1. A video for this is included as supplementary material. ${ }^{29}$

Measurements were performed on four samples: a 1$\mathrm{mm}$ thick duralumin plate, a $1.5-\mathrm{mm}$ thick fused quartz plate, a $0.8-\mathrm{mm}$ thick silicon wafer, and a 1-mm thick titanium plate. Reference thicknesses were determined using a digital caliper (accuracy of $0.01 \mathrm{~mm}$ ). The transversal dimensions of the samples were larger than $50 \mathrm{~mm}$, allowing the modes to travel along the whole probe array before being reflected at the plate boundaries. Table I summarizes the nominal values for the elastic coefficients, the mass density and the reference thickness of the samples.

\section{B. Estimation of material properties and symmetry axes}

In the following, we introduce the forward waveguide model used to analyze the elastic wave propagation in anisotropic plates and we shortly recall the main constituent parts

TABLE I. Reference values.

\begin{tabular}{|c|c|c|c|c|c|c|c|}
\hline & \multicolumn{7}{|c|}{ Reference values } \\
\hline & \multicolumn{5}{|c|}{$\begin{array}{l}\text { Elastic coefficients } \\
\qquad(\mathrm{GPa})\end{array}$} & \multirow{2}{*}{$\begin{array}{c}\text { Density } \\
\left(\mathrm{g} \mathrm{cm}^{-3}\right) \\
\rho\end{array}$} & \multirow{2}{*}{$\begin{array}{c}\text { Thickness } \\
\text { (mm) } \\
d\end{array}$} \\
\hline & $c_{11}$ & $c_{12}$ & $c_{33}$ & $c_{13}$ & $c_{44}$ & & \\
\hline Duralumin $^{\mathrm{a}}$ & 114.4 & - & - & - & 27.2 & 2.795 & 0.992 \\
\hline Fused quartz ${ }^{\mathrm{a}}$ & 78.4 & - & - & - & 31.2 & 2.200 & 1.475 \\
\hline Silicon $^{\mathrm{b}}$ & 165.6 & 63.9 & - & - & 79.5 & 2.329 & 0.779 \\
\hline Titanium $^{c}$ & 162.4 & 92.0 & 180.7 & 69.0 & 46.7 & 4.506 & 1.012 \\
\hline
\end{tabular}

${ }^{\text {a} B r i g g s ~ a n d ~ K o l o s o v ~(R e f . ~ 30) . ~}$

${ }^{\mathrm{b}}$ Prada et al. (Ref. 31).

${ }^{\mathrm{c}}$ Royer et al. (Ref. 32). 


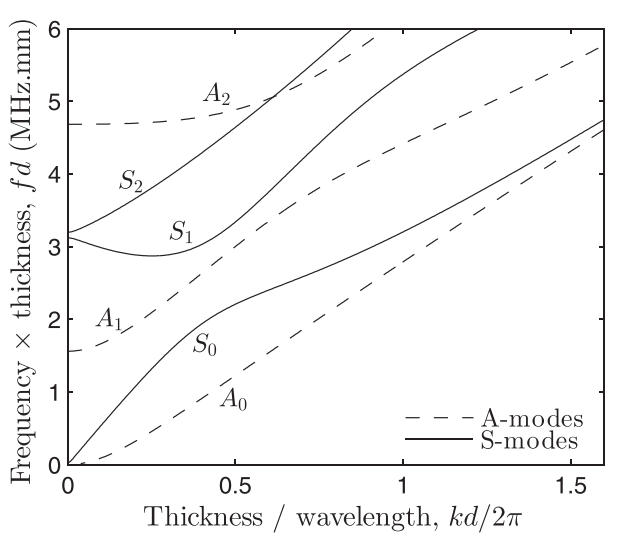

(a) Duralumin plate

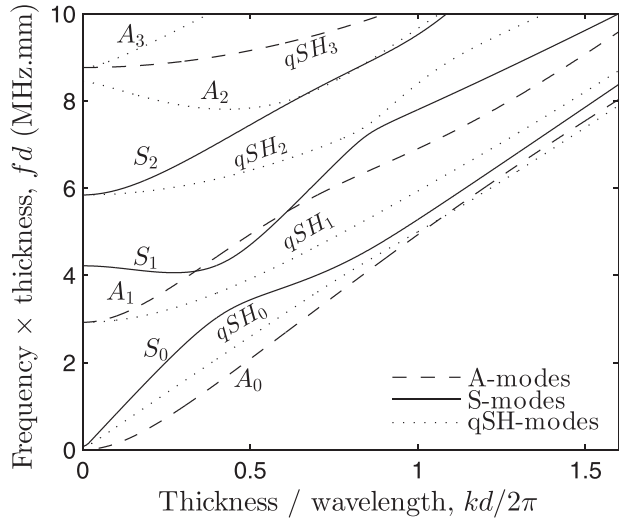

(b) Silicon wafer
FIG. 2. Dispersion curves in the (kd, fd)-plane for guided waves propagating in (a) an isotropic duralumin plate and (b) a silicon wafer along an oblique axis (i.e., $\phi=22.5^{\circ}$ ). The modes are labeled according to Royer et al. (Ref. 36). of the inverse problem, that are (i) the definition of an objective function as a metric to compare measured and predicted guided modes and (ii) the use of an algorithm to iteratively optimize the objective function and infer the optimal model parameters.

\section{Forward problem}

The dispersion equations for elastic wave propagation in anisotropic plates of monoclinic or higher symmetry were implemented according to the numerical procedure described by Li and Thompson, ${ }^{33}$ which establishes the relation between the material properties and the corresponding guided waves by partial wave theory. Rayleigh-Lamb dispersion relations were derived for the specific cases of (1) isotropic (two independent elastic coefficients), (2) cubic (three independent elastic coefficients), and (3) transversely isotropic (five independent elastic coefficients) materials. The adopted formulation allowed accounting for propagation along a material symmetry axis as well as propagation in an arbitrary direction.

We consider a homogeneous free plate of thickness $d$ possessing at least a monoclinic symmetry with respect to the $x_{2} x_{3}$-plane, as depicted in Fig. 1(b). By solving Christoffel equations under consideration of stress-free boundary conditions, the solutions of the corresponding dispersion equation can be expressed as guided modes in the $(k, f)$-domain, which are determined by the thickness $d$, mass density $\rho$, stiffness coefficients $c_{i j}(i, j=1, \ldots, 6)$ and propagation direction $\phi$ according to the principal symmetry axes of the material. ${ }^{33}$ Because the plate possesses a symmetry plane normal to the $x_{1}$-direction, solutions can always be separated into symmetric (S) and antisymmetric (A) modes. Hence, the dispersion equation accounting for each family of modes can be stated as

$$
\Omega_{M}(k, f ; \boldsymbol{\theta})=0, \quad \text { with } \quad M=A \quad \text { or } S,
$$

where the vector $\boldsymbol{\theta}=\left[d c_{i j} \rho \phi\right]$ denotes the model parameters. In practice, the dispersion equation can be formulated as a function of the bulk wave velocities and stiffness ratios, with the mass density being included in the velocities. ${ }^{34}$ For isotropic, cubic or transversely isotropic materials, additional symmetry planes introduce significant simplifications in the dispersion equation. Moreover, when the wave propagates in a direction lying in a symmetry plane, partial waves polarized in the sagittal plane become decoupled from the shear horizontally polarized waves. In such a case, the characteristic equation factorizes to a second-order polynomial. For example, Fig. 2 depicts the guided modes for an isotropic duralumin plate and for a silicon wafer with cubic symmetry (propagation along an oblique axis, with an angle $\phi=22.5^{\circ}$ ). As can be observed, shear horizontal ( $\mathrm{SH}$ ) modes that exist in planes of symmetry degenerate into quasi-SH modes, denoted here by qSH, if the sagittal plane is a non-principal symmetry plane of the material. ${ }^{35}$

\section{Inverse problem}

Generally, the difference between experimental and modeled guided modes is the most important constituent part of the objective function, in which the inversion process can be regarded as curve fitting (i.e., minimization of an Euclidean distance, $F_{\mathrm{LS}}(\boldsymbol{\theta})$, in a least-squares sense), for instance,

$$
F_{L S}(\boldsymbol{\theta})=\sum_{m=1}^{M} \sum_{i=1}^{I_{m}}\left(f^{m}\left(k_{i}\right)-f^{m}\left(k_{i} ; \boldsymbol{\theta}\right)\right)^{2},
$$

where $f^{m}\left(k_{i}\right)$ and $f^{m}\left(k_{i} ; \boldsymbol{\theta}\right)$ are the measured and modeled frequencies for a given mode $m$ at a wave number $k_{i}$ $\left(i=1, \ldots, I_{m}\right) .{ }^{23,37}$ Such approach is robust but requires, for each $\boldsymbol{\theta}$, the explicit computation of the theoretical guided modes using an iterative root-finding algorithm (e.g., Muller or Newton-Raphson) and prior identification of the modes. Depending on the complexity of the inspected medium, this computation can be time-consuming (i.e., typically a few seconds as small frequency steps are needed for numerically inferring the roots of the dispersion equation) and can even lead to numerical errors when two roots are in close proximity, which is typically the case in the vicinity of crossing points, ${ }^{38}$ thus requiring further numerical refinements. ${ }^{39}$ Alternatively, few authors proposed to directly use the dispersion equation as objective function $F_{D}(\boldsymbol{\theta}){ }^{16,19}$ The idea is to evaluate Eq. (1) on the experimental data $\left(k_{n}, f_{n}\right)$, with $n=1, \ldots, N$,

$$
F_{D}(\boldsymbol{\theta})=\sum_{\mathrm{n}=1}^{\mathrm{N}} \log \left(\left|\Omega_{A}\left(k_{n}, f_{n} ; \boldsymbol{\theta}\right) \times \Omega_{S}\left(k_{n}, f_{n} ; \boldsymbol{\theta}\right)\right|\right) .
$$


In such a case, solving the inverse problem consists in finding the optimal model parameters $\hat{\boldsymbol{\theta}}$ that minimize the function defined by Eq. (3). Unfortunately, this formulation is not convenient, because the dynamics of the dispersion equation is inhomogeneous in the $(k, f)$-plane [Fig. 3(a)]. In contrast, calculating the sign of
$\Omega_{A}(k, f ; \boldsymbol{\theta}) \times \Omega_{S}(k, f ; \boldsymbol{\theta})$ delivers a function [Fig. 3(b)], whose discontinuities can directly be associated with the guided modes.

To evaluate whether or not an experimental data point $\left(k_{n}, f_{n}\right)$ is a solution of the dispersion equation, we introduce the function

$$
G_{M}\left(k_{n}, f_{n} ; \boldsymbol{\theta}\right)=\frac{1}{2}\left(3-\left|\sum_{j=-1}^{1} \operatorname{sgn}\left(\Omega_{M}\left(k_{n}, f_{n}+j \Delta f ; \boldsymbol{\theta}\right)\right)\right|\right)= \begin{cases}1 & \text { if }\left(k_{n}, f_{n}\right) \text { is a solution to Eq. (1) } \\ 0 & \text { otherwise }\end{cases}
$$

with $M=A$ or $S$, where $\Delta f$ is set to half the frequency step used in the temporal Fourier transform of the data. This simple function allows us to track the sign changes of $\Omega_{M}(k, f ; \boldsymbol{\theta})$, with $M=A$ or $S$, in the vicinity of an experimental data point $\left(k_{n}, f_{n}\right)$, i.e., between $\left(k_{n}, f_{n}-\Delta f\right)$ and $\left(k_{n}, f_{n}+\Delta f\right)$, as depicted in Fig. $3(b)$. Note that this finite difference is evaluated with respect to $f$ (and not to $k$ ), because the slope $\Delta f / \Delta k$, which approximates the group velocity, is always finite and smaller than the longitudinal bulk wave velocity, whereas around zerogroup velocity points (e.g., cut-off frequencies and zerogroup velocity resonances $\left.{ }^{40}\right) \Delta f / \Delta k$ diverges. The objective function can thus be written as

$$
F(\boldsymbol{\theta})=\sum_{n=1}^{N} w_{n}\left(G_{A}\left(k_{n}, f_{n} ; \boldsymbol{\theta}\right)+G_{S}\left(k_{n}, f_{n} ; \boldsymbol{\theta}\right)\right),
$$

where $w_{n}$ is the weighting factor introduced in Fig. 1. Solving the inverse problem now consists in maximizing the objective function $F(\boldsymbol{\theta})$, that is to maximize the number of experimental data points that fulfill the dispersion equation. To serve as an example, Fig. 4(a) depicts the underlying idea of Eq. (5), where experimental data points $\left(k_{n}, f_{n}\right)$ that contribute to the objective function $F(\boldsymbol{\theta})$ are displayed as colored dots according to their weight $w_{n}$, while those that do not contribute to $F(\boldsymbol{\theta})$ are displayed in light gray color. For sake of clarity, in the following, we will use the traditional representation depicted in Fig. 4(b) to display the results. This objective function $F(\boldsymbol{\theta})$ presents several advantages for multimodal inversion of guided waves: computational costs are drastically reduced by avoiding the numerical calculation of the theoretical dispersion curves, as the dispersion equation is only evaluated on $3 \times N$ experimental data and the identification of experimental modes prior to the inversion process is not required. Formally, the optimal model parameters $\hat{\boldsymbol{\theta}}$ result from

$$
\hat{\boldsymbol{\theta}}=\arg \max _{\boldsymbol{\theta} \in \Theta} F(\boldsymbol{\theta}),
$$

where $\Theta$ denote the bounds of the model parameters $\boldsymbol{\theta}$. Genetic algorithms are applied to solve Eq. (6), because of their ability in finding a near global solution for non-convex multidimensional objective function, without the need of an accurate initial guess for the model parameters. ${ }^{28}$

A large searching domain was chosen by setting the bounds for the elastic coefficients $c_{i j}$ to $[0.61 .4] \times$ the nominal values reported in Table I and those for the thickness $d$ to $\pm 20 \%$ the reference thickness measured by a caliper. For material with unknown symmetry axis, the bounds for the propagation direction angle $\phi$ were set to $[0 \pi / 2-\epsilon]$ for cubic symmetry and $[0 \pi-\epsilon]$ for transverse isotropic symmetry, where $\epsilon \ll \pi / 180$ represents a small angle used to avoid periodic-equivalent solutions due to material symmetry. To ensure physically acceptable solutions,

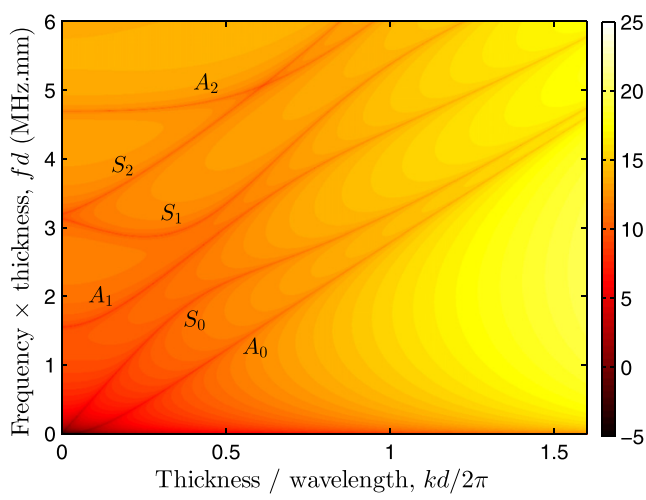

(a) $\log \left(\left|\Omega_{A}(k, f ; \theta) \times \Omega_{S}(k, f ; \theta)\right|\right)$

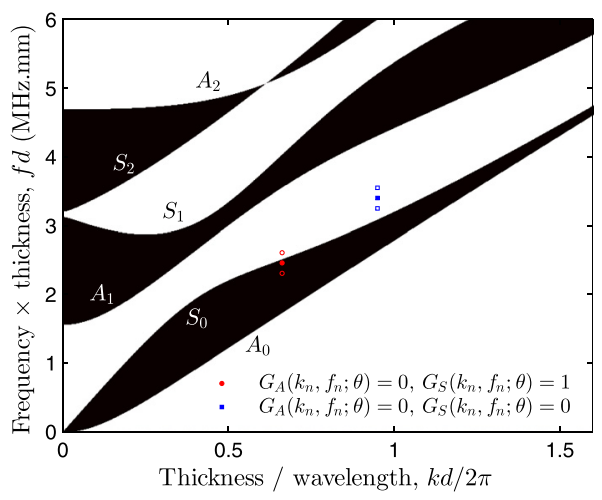

(b) $\operatorname{sgn}\left(\Omega_{A}(k, f ; \theta) \times \Omega_{S}(k, f ; \theta)\right)$
FIG. 3. (Color online) (a) Map of the values of $\log \left(\mid \Omega_{A}(k, f ; \boldsymbol{\theta}) \times\right.$ $\left.\Omega_{S}(k, f ; \boldsymbol{\theta}) \mid\right)$ obtained by sweeping the wave number $k$ and frequency $f$, whose positions of the loci display the dispersion curves; (b) binary map obtained by computing the sign of $\Omega_{A}(k, f ; \boldsymbol{\theta}) \times \Omega_{S}(k, f ; \boldsymbol{\theta})$, whose sign changes on the dispersion curves. Solid symbols stand for experimental data points $\left(k_{n}, f_{n}\right)$, whereas hollow symbols stand for their two neighbours, i.e., $\left(k_{n}, f_{n}-\Delta f\right)$ and $\left(k_{n}\right.$, $f_{n}+\Delta f$ ), used to track the sign change of $\Omega_{M}(k, f ; \boldsymbol{\theta})$, with $M=A$ or $S$. 


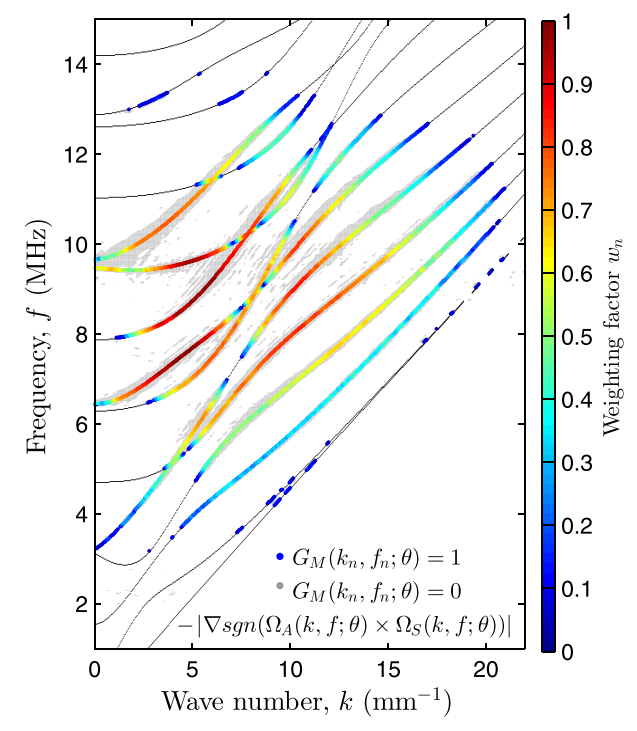

(a) Computation of the objective function $F(\theta)$

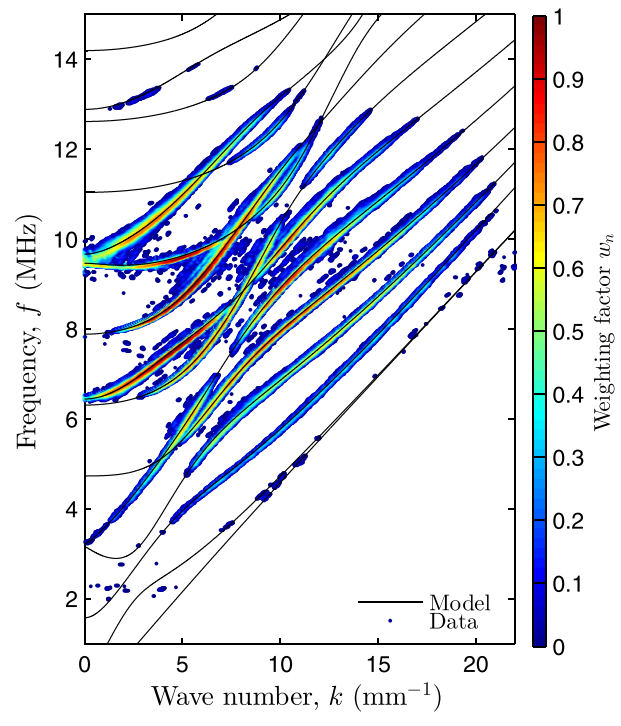

(b) Equivalent representation
FIG. 4. (Color online) Computation of the objective function $F(\boldsymbol{\theta})$ : (a) Experimental data points $\left(k_{n}, f_{n}\right)$ that contribute to the objective function are displayed as colored dots according to their weight $w_{n}$, while points that do not contribute to the objective function are displayed in light gray color; (b) equivalent representation: Points of the Fourier transformed data that are used in the inversion, along with the theoretical dispersion curves obtained with the estimated model parameters. thermodynamics constraints requiring the elasticity tensor to be positive-definite were adopted. ${ }^{41}$ For the isotropic, cubic and transverse isotropic cases, this, respectively, lead to $\left\{c_{11}>\frac{4}{3} c_{44}>0\right\},\left\{c_{11}>c_{12}>0 ; c_{44}>0\right\}$, and $\left\{c_{11}>c_{66}\right.$ $\left.>0 ; c_{44}>0 ; c_{33}\left(c_{11}-c_{66}\right)>c_{13}^{2}\right\}$. For all tests, the inverse procedure was solved using a random initial population of $N_{p}=20$ chromosomes within the genetic algorithms. The number of generations $N_{g}$ was adaptive in the sense that the algorithm stopped if the optimal solution $\hat{\boldsymbol{\theta}}$ remained unchanged along 50 generations. The empirical variables $N_{p}$ and $N_{g}$, along with the probabilities of the genetic operators, were set so that the convergence to a near global optimum was guaranteed, while establishing a trade-off between inverse problem errors and computational cost. ${ }^{42}$ For a comprehensive description of the genetic algorithms-based inversion of multimode guided waves, the reader is referred to our former paper. ${ }^{43}$

\section{RESULTS}

To evaluate the performance of the proposed inversion procedure, two case studies were investigated. First, we assumed that the propagation direction $\phi$ (i.e., angle between the probe and material principal symmetry axes) was known, and the inverse procedure was applied to infer the elastic coefficients $c_{i j}$ and the plate thickness $d$ of the tested materials. Second, for anisotropic plates of known thickness $d$, the inverse procedure was applied to infer the elastic coefficients $c_{i j}$ and propagation direction $\phi$.

\section{A. Case study I}

Four different plates are considered, for which the propagation direction $\phi$ is supposed to be known. The results obtained for the isotropic duralumin and fused quartz plates are presented in Fig. 5. The optimal matching between the experimental data and the estimated Lamb modes shows a remarkable agreement.
For the silicon wafer, the results obtained for propagation along the $\left[\begin{array}{lll}0 & 1 & 1\end{array}\right]$ symmetry axis (i.e., $\phi=45^{\circ}$ ) and for an oblique propagation (i.e., $\phi=65^{\circ}$ ) are presented in Fig. 6. The optimal matching between the experimental data and the estimated guided modes shows again a good agreement. If the plane of propagation coincides with a plane of symmetry [Fig. 6(a)], the probe only detects pure Lamb modes, while, if the sagittal plane is not a plane of symmetry [Fig. 6(b)], then, in addition to quasi-Lamb modes, quasi-SH modes having a non-zero normal displacement are also detected (e.g., $\mathrm{qSH}_{1}$ between 5 and $8 \mathrm{MHz}$, and $\mathrm{qSH}_{2}$ between 9 and $12 \mathrm{MHz}) .{ }^{35}$ In both cases, the multiparametric inversion method delivers estimates $\hat{\boldsymbol{\theta}}$ that are in close agreement, with an absolute difference lower than $1.5 \mathrm{GPa}$ for the elastic coefficients and lower than $2 \mu \mathrm{m}$ for the thickness.

Last, results obtained for the titanium plate are presented in Fig. 7. For this transverse isotropic material, the concurrent inference of all model parameters $\boldsymbol{\theta}$ is particularly challenging, as the multiparametric inverse problem involves five independent elastic coefficients and the plate thickness. In such a case, it is useful to take advantage of two measurements on different symmetry planes of propagation, thus reducing the dimensionality of the searching domain $\Theta$. Hence, if the first propagation plane coincides with the isotropy plane of symmetry (i.e., $x_{1} x_{2}$-plane, $\phi=0^{\circ}$ ), a first set of model parameters leads to $\boldsymbol{\theta}_{1}=\left[c_{11} c_{12} d\right]$. Then, if the second propagation plane coincides with the transverse isotropic plane of symmetry (i.e., $x_{1} x_{3}$-plane, $\phi=90^{\circ}$ ), a second set of model parameters leads to $\boldsymbol{\theta}_{2}=\left[c_{11} c_{33} c_{13} c_{44} d\right]$. Consequently, the initial six-parameter inverse problem can be reduced to two problems with three unknown model parameters each [Figs. 7(a) and 7(b)].

Even more interesting, the multiparametric inverse problem can also be solved using a single measurement along a non-principal symmetry axis [e.g., $\phi=45^{\circ}$, see Fig. 7(c)], for which the dispersion equation depends on all elastic coefficients (i.e., $\boldsymbol{\theta}=\left[c_{11} c_{12} c_{33} c_{13} c_{44} d\right]$ ). For both approaches, the multiparametric inverse problem delivers estimates $\hat{\boldsymbol{\theta}}$ that are in close agreement with an absolute 


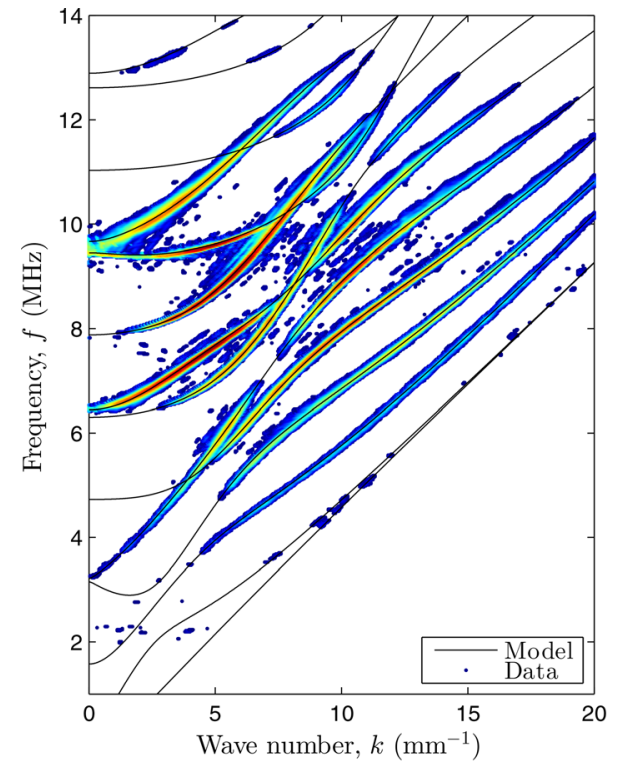

(a) $\hat{\theta}=[113.5 \mathrm{GPa} ; 27.2 \mathrm{GPa} ; 0.989 \mathrm{~mm}]$

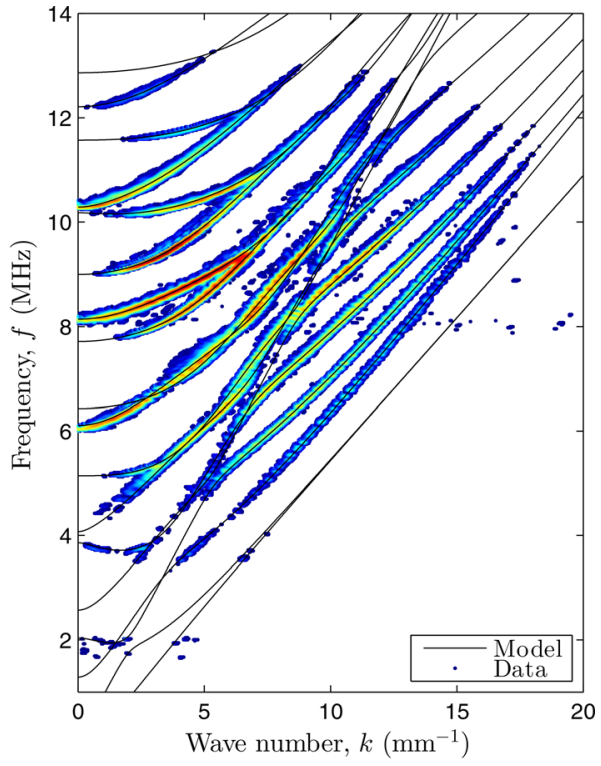

(b) $\hat{\theta}=[78.8 \mathrm{GPa} ; 31.4 \mathrm{GPa} ; 1.470 \mathrm{~mm}]$

FIG. 5. (Color online) Optimal matching between the experimental data (color dots) and the Lamb modes (continuous lines) for the isotropic plates: (a) 1-mm thick duralumin plate and (b) 1.5 -mm thick fused quartz plate.

difference lower than $3 \mathrm{GPa}$ for the elastic coefficients and lower than $3 \mu \mathrm{m}$ for the plate thickness. However, as discussed below in Table II, the inverse problem errors associated with a searching domain $\Theta$ of higher dimensionality are typically larger. Again, for the case where the plane of propagation does not coincide with a plane of symmetry [Fig. 7(c)], quasi-SH modes that have a non-zero normal displacement are also detected in addition to quasi-Lamb modes (e.g., $\mathrm{qSH}_{5}$ between 6.5 and $7.5 \mathrm{MHz}, \mathrm{qSH}_{6}$ between 8 and $9 \mathrm{MHz}, \mathrm{qSH}_{7}$ between 9.5 and $12 \mathrm{MHz}$, and $\mathrm{qSH}_{8}$ between 11 and $12 \mathrm{MHz}$ ).

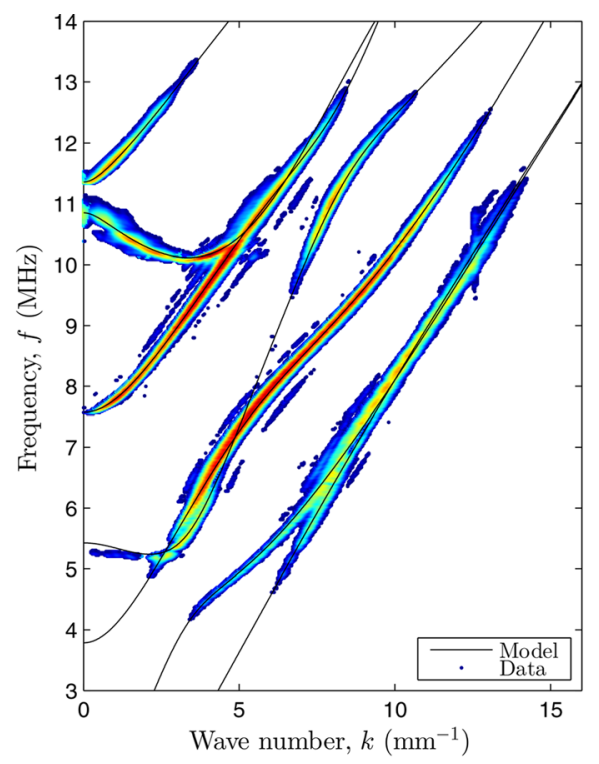

(a) $\hat{\theta}=[164.7 \mathrm{GPa} ; 63.0 \mathrm{GPa} ; 80.2 \mathrm{GPa} ; 0.775 \mathrm{~mm}]$

\section{B. Case study II}

The propagation direction $\phi$ is now supposed to be unknown, but the thickness is assumed to be known and equal to the values found in case study I. Results obtained for the silicon wafer for propagation along the $\left[\begin{array}{lll}0 & 1 & 0\end{array}\right]$ symmetry axis $\left(\phi=0^{\circ}\right)$ and for a propagation direction $\phi=75^{\circ}$ are presented in Figs. 8(a) and 8(b). As can be observed, the propagation angle $\phi$ is correctly estimated with an absolute error lower than $1.5^{\circ}$. For both angles, the multiparametric inversion delivers estimates $\hat{\boldsymbol{\theta}}$ that are in close agreement, with an absolute difference lower than $3 \mathrm{GPa}$ for the elastic

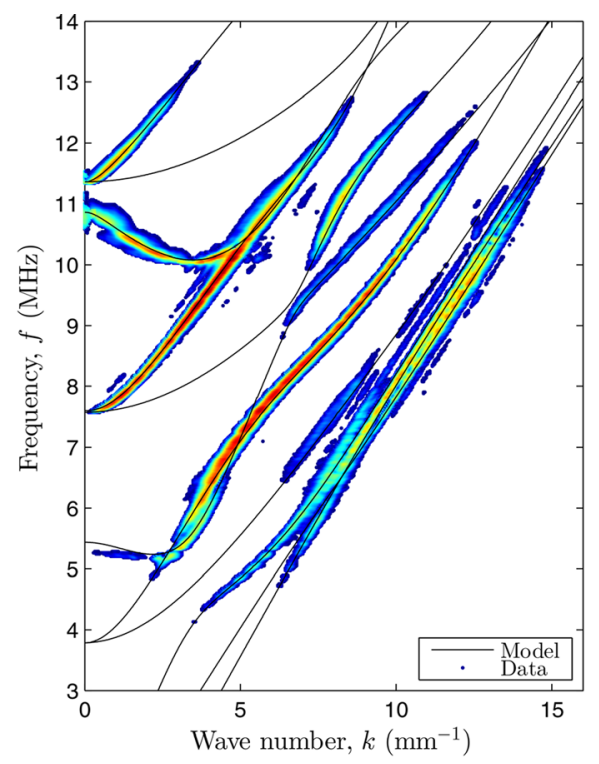

(b) $\hat{\theta}=[164.6 \mathrm{GPa} ; 64.3 \mathrm{GPa} ; 80.1 \mathrm{GPa} ; 0.774 \mathrm{~mm}]$

FIG. 6. (Color online) Optimal matching between experimental data (color dots) and guided modes (continuous lines) for the silicon wafer for propagation (a) along the $\left[\begin{array}{lll}0 & 1 & 1\end{array}\right]$ symmetry axis $\left(\phi=45^{\circ}\right)$ and (b) for an oblique axis $\left(\phi=65^{\circ}\right)$. 


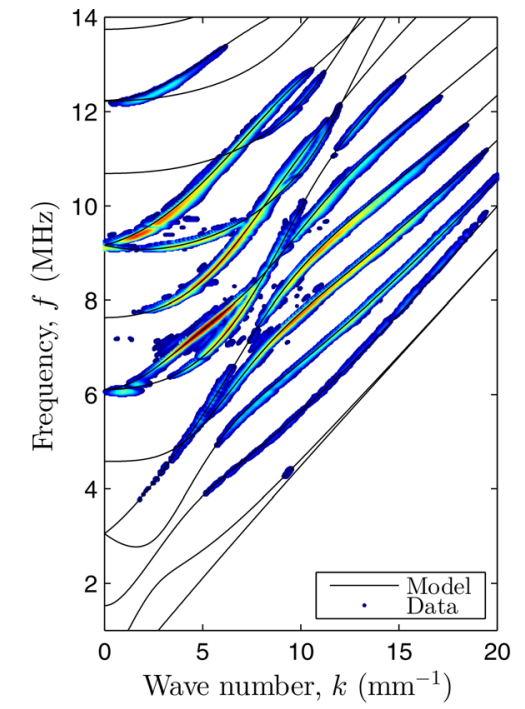

(a) $\hat{\theta}_{1}=[169.1 \mathrm{GPa} ; 84.8 \mathrm{GPa} ; 1.002 \mathrm{~mm}]$

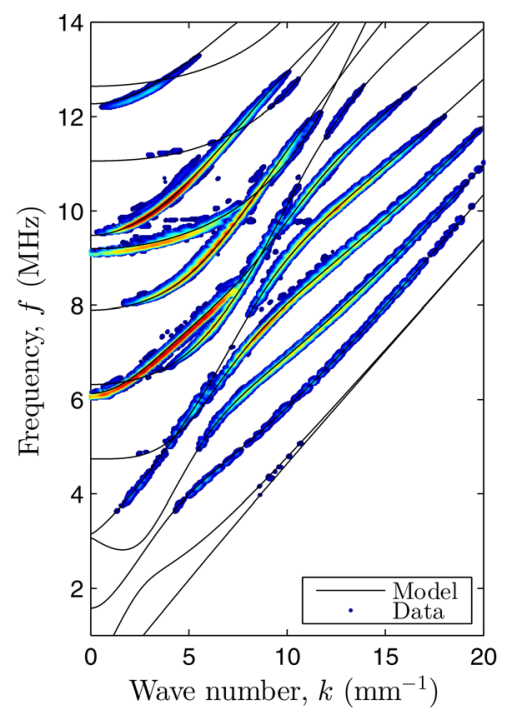

(b) $\hat{\theta}_{2}=[173.2 \mathrm{GPa} ; 81.0 \mathrm{GPa} ; 45.2 \mathrm{GPa}]$

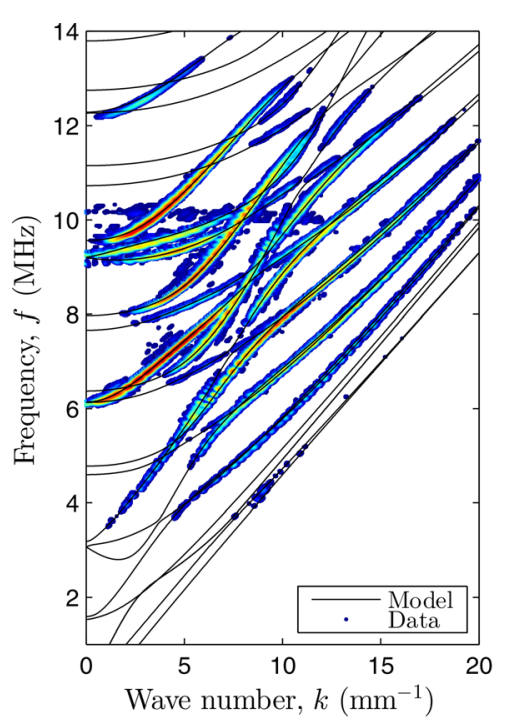

(c) $\hat{\theta}=[168.9 \mathrm{GPa} ; 83.7 \mathrm{GPa} ; 173.1 \mathrm{GPa}$ : 84.1 GPa; $46.8 \mathrm{GPa} ; 1.005 \mathrm{~mm}]$

FIG. 7. (Color online) Optimal matching between experimental data (color dots) and guided modes (continuous lines) for the titanium plate and for propagation (a) along the $\left[\begin{array}{lll}0 & 1 & 0\end{array}\right]$ symmetry axis (i.e., $\phi=0^{\circ}$; plane of isotropy); (b) along the $\left[\begin{array}{lll}0 & 0 & 1\end{array}\right]$ symmetry axis (i.e., $\phi=90^{\circ}$; plane of transverse isotropy); and (c) out-of-symmetry axis (i.e., $\phi=45^{\circ}$ ).

coefficients. Note that these estimates are also in close agreement with those depicted in Fig. 6.

Results obtained for the titanium plate along an oblique axis (i.e., $\phi=33^{\circ}$ ) are presented in Fig. 8(c). Again, the retrieved propagation angle $\phi$ is in good agreement with the expected one. The inversion method delivers estimates $\hat{\boldsymbol{\theta}}$ that are also in close agreement with those obtained in the first case study (Fig. 7).

\section{DISCUSSION}

As often required for industrial applications, single-side access to the tested plates is satisfied by the proposed experimental technique. The measured dispersion curves contain numerous higher-order modes, which makes a precise inversion of the model parameters $\boldsymbol{\theta}$ possible. For all the tested samples, the reported estimates are in excellent agreement with the reference values from Table I. It is noteworthy that the acquisition of a single set of dispersion curves can be performed in real-time $(\sim 0.01 \mathrm{~s})$, which represents an appealing aspect compared to other experimental techniques where numerous measurements are required. In addition, the main advantage of the proposed inversion procedure is that it drastically reduces the computational costs, because the calculation of the theoretical dispersion curves is not required. For a given set of model parameters $\boldsymbol{\theta}$, the forward calculation for wave propagation along a principal symmetry axis (i.e., characteristic equation is a second-order polynomial) and an oblique axis (i.e., third-order polynomial) approximately takes $\sim 0.01$ and $\sim 0.04 \mathrm{~s}$, respectively. In contrast, numerical iterative rootfinding algorithms used for the calculation of the dispersion curves last around a few seconds (i.e., a factor of 200) ${ }^{18}$ and are prone to numerical errors. ${ }^{38}$ All computations were performed using Matlab ${ }^{\circledR}$ on a standard desktop computer (Intel Xeon CPU E5-2623 v3@3.00 GHz). For isotropic materials, convergence to a near global solution was typically achieved

TABLE II. Optimal model parameters for the investigated samples (the parameters in bold were kept constant within the inverse procedure).

\begin{tabular}{|c|c|c|c|c|c|c|c|}
\hline & \multicolumn{7}{|c|}{ Optimal model parameters $\hat{\boldsymbol{\theta}}$} \\
\hline & \multicolumn{5}{|c|}{ Elastic coefficients (GPa) } & \multirow{2}{*}{$\begin{array}{c}\text { Thickness (mm) } \\
d\end{array}$} & \multirow{2}{*}{$\begin{array}{c}\text { Angle }\left(^{\circ}\right) \\
\phi\end{array}$} \\
\hline & $c_{11}$ & $c_{12}$ & $c_{33}$ & $c_{13}$ & $c_{44}$ & & \\
\hline \multirow{2}{*}{ Duralumin } & $113.0 \pm 1.2$ & - & - & - & $27.2 \pm 0.2$ & $0.988 \pm 0.006$ & $\mathbf{0}$ \\
\hline & $112.6 \pm 1.4$ & - & - & - & $27.1 \pm 0.3$ & $0.986 \pm 0.007$ & [0:5:180] \\
\hline \multirow{2}{*}{ Quartz } & $79.0 \pm 1.1$ & - & - & - & $31.5 \pm 0.3$ & $1.472 \pm 0.010$ & $\mathbf{0}$ \\
\hline & $79.4 \pm 1.2$ & - & - & - & $31.7 \pm 0.3$ & $1.476 \pm 0.010$ & [0:5:180] \\
\hline \multirow{3}{*}{ Silicon } & $164.0 \pm 4.8$ & $61.9 \pm 4.3$ & - & - & $80.2 \pm 1.3$ & $0.775 \pm 0.008$ & 45 \\
\hline & $161.9 \pm 4.6$ & $60.4 \pm 4.8$ & - & - & $80.6 \pm 1.0$ & 0.775 & $87.5 \pm 7.6$ \\
\hline & $163.8 \pm 6.5$ & $61.7 \pm 5.6$ & - & - & $80.2 \pm 2.1$ & $0.774 \pm 0.012$ & {$[0: 5: 180]$} \\
\hline \multirow{4}{*}{ Titanium } & $168.9 \pm 2.2$ & $85.1 \pm 1.6$ & $171.8 \pm 3.6$ & $80.2 \pm 1.9$ & $45.4 \pm 0.4$ & $1.000 \pm 0.008$ & {$[0,90]$} \\
\hline & $169.0 \pm 3.1$ & $82.4 \pm 2.1$ & $175.0 \pm 5.0$ & $86.3 \pm 3.4$ & $46.6 \pm 1.0$ & $1.007 \pm 0.011$ & 45 \\
\hline & $165.7 \pm 6.5$ & $80.4 \pm 2.6$ & $171.5 \pm 2.2$ & $84.7 \pm 2.9$ & $45.5 \pm 0.9$ & 1.004 & $35.1 \pm 4.3$ \\
\hline & $164.1 \pm 9.4$ & $77.1 \pm 4.9$ & $173.8 \pm 6.3$ & $82.3 \pm 5.5$ & $45.3 \pm 1.7$ & $1.009 \pm 0.014$ & [0:5:180] \\
\hline
\end{tabular}




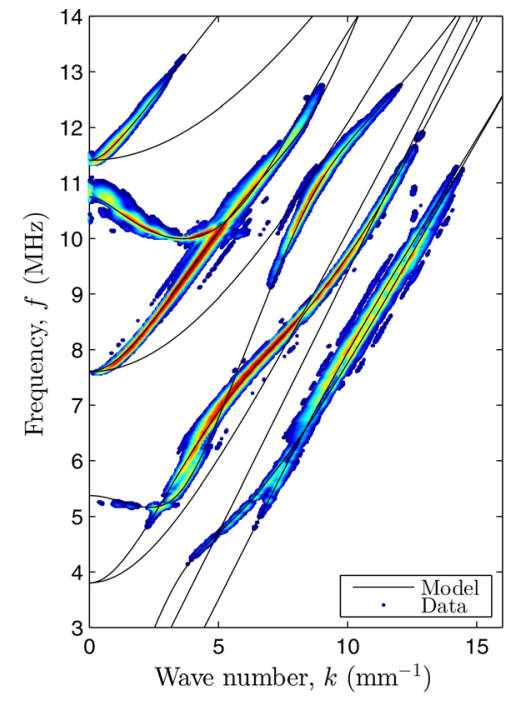

(a) $\hat{\theta}=\left[161.6 \mathrm{GPa} ; 59.2 \mathrm{GPa} ; 80.9 \mathrm{GPa} ; 88.8^{\circ}\right]$

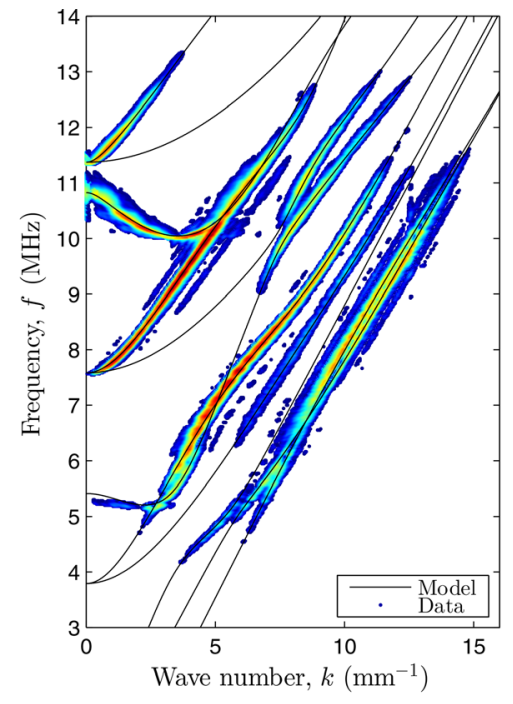

(b) $\hat{\theta}=\left[163.9 \mathrm{GPa} ; 62.1 \mathrm{GPa} ; 80.4 \mathrm{GPa} ; 74.5^{\circ}\right]$

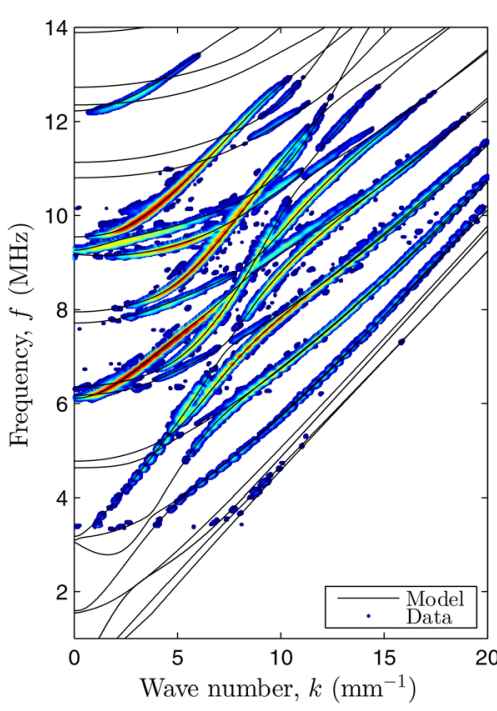

(c) $\hat{\theta}=[168.1 \mathrm{GPa} ; 83.1 \mathrm{GPa} ; 169.8 \mathrm{GPa}$; 86.5 GPa; $\left.46.0 \mathrm{GPa} ; 34.3^{\circ}\right]$

FIG. 8. (Color online) Optimal matching between experimental data (color dots) and guided modes (continuous lines): for the silicon wafer for propagation (a) along the $\left[\begin{array}{lll}0 & 1 & 0\end{array}\right]$ symmetry axis $\left(\phi=0^{\circ}\right)$ and (b) out-of-symmetry axis $\left(\phi=75^{\circ}\right)$; and (c) for the titanium plate for an oblique propagation axis $\left(\phi=33^{\circ}\right)$.

after $N_{g}=200$ generations, thus leading to a computation time of approximately $40 \mathrm{~s}$. For anisotropic materials, the convergence rate is somewhat slower (i.e., $N_{g}=300$ ), yielding computation times of $60 \mathrm{~s}$ for propagation along a principal symmetry axis and $240 \mathrm{~s}$ for an oblique axis.

In order to test the statistical stability of the optimization algorithm, ${ }^{16}$ the inversion procedure was run 36 times on the same measurement for each sample. Then, to evaluate the uncertainty due to measurements variability, the procedure was run on 36 measurements (acquired each $5^{\circ}$ between 0 and $180^{\circ}$ ). Results (mean \pm standard deviation) obtained for each sample are listed in Table II.

As can be observed, the errors on the estimated material properties increase with the number of model parameters. Moreover, the uncertainties due to measurements variability given in the last rows are generally larger than the inversion errors. This was expected since measurement uncertainties cannot be separated from the uncertainties due to the reconstruction algorithm. For the isotropic plates (i.e., duralumin and fused quartz), the inversion errors are lower than $2 \%$ and $1 \%$ for the longitudinal and transverse elastic coefficients, respectively, and lower than $1 \%$ for the plate thickness. For the silicon wafer, the inversion errors are lower than $3 \%$, $8 \%$, and $2 \%$ for the longitudinal, off-diagonal and transverse elastic coefficients, respectively, around $1 \%$ for the plate thickness, and lower than $9 \%$ for the propagation angle. For the titanium plate, when the inverse problem is solved in two steps, the errors are lower than $2 \%, 2 \%$, and $1 \%$ for the longitudinal, off-diagonal and transverse elastic coefficients, respectively, and around $1 \%$ for the plate thickness. When the inverse problem is solved in one step, errors tend to increase, and reach $12 \%$ for the angle of propagation.

For anisotropic materials, i.e., silicon wafer and titanium plate, the larger errors reported for the off-diagonal elastic coefficients can be explained as follows: it has been shown that they have little influence on the dispersion behavior and are therefore difficult to measure accurately. In addition, they are generally small compared to the other elastic coefficients and ambiguous to differentiate. ${ }^{11}$ In contrast, the larger errors reported for the angle of propagation may be partly caused by a slight misalignment between the probe and the principal symmetry axis of the material at the initial stage of the measurements (accuracy of $1^{\circ}$ ). As a further source of error, it should be noted that the Fourier coefficients (i.e., $w_{n}$ ) corresponding to the qSH-modes are typically lower than that of quasi-Lamb modes, and thus contribute to a smaller extent to the objective function, which could in turn lead to modes misidentification or biased elastic coefficients in the inverse procedure.

\section{CONCLUSION}

This paper reported on the assessment of material properties from elastic guided wave measurements on anisotropic plates. Experiments were performed using a linear piezoelectric transducer array, which allowed the real-time measurements of many guided modes in arbitrary directions. The proposed reconstruction procedure using genetic algorithms was proven feasible to identify the full set of anisotropic elastic coefficients and either the plate thickness or the propagation direction from a single out-of-symmetry axis measurement. For all the tested samples, the reported estimates were in excellent agreement with reference values from the literature. The model-based inverse problem solution presents several advantages: (1) it requires minimal data processing and allows higher-order modes to be taken into account without any prior identification and (2) it avoids the numerical resolution of the dispersion equation. This results in a very low computational cost, thus opening perspectives towards real-time material characterization and defect detection. Future works will extend this study to layered (composite) structures. To this end, signal-to-noise ratio enhancement could be achieved by applying a singular value decomposition to the complete inter-element array response matrix. We will also investigate the feasibility of exploiting 
targeted data of the dispersion spectrum, such as cut-off frequencies and zero-group velocity modes, that exhibit a particular sensitivity to one or another model parameter, in order to speed up the inversion process.

\section{ACKNOWLEDGMENTS}

This work was supported by the Swiss National Science Foundation (Advanced Postdoc Mobility Fellowship P300P2_174481) and the Chaire SAFRAN ESPCI, Paris.

${ }^{1}$ C. Aristégui and S. Baste, "Optimal recovery of the elasticity tensor of general anisotropic materials from ultrasonic velocity data," J. Acoust. Soc. Am. 101(2), 813-833 (1997).

${ }^{2}$ C. Aristégui and S. Baste, "Optimal determination of the material symmetry axes and associated elasticity tensor from ultrasonic velocity data," J. Acoust. Soc. Am. 102(3), 1503-1521 (1997).

${ }^{3}$ B. Castagnede, K. Kim, W. Sachse, and M. Thompson, "Determination of the elastic constants of anisotropic materials using laser-generated ultrasonic signals," J. Appl. Phys. 70(1), 150-157 (1991).

${ }^{4}$ A. Nayfeh, Wave Propagation in Layered Anisotropic Media: With Application to Composites (Elsevier, Amsterdam, 1995), Vol. 39.

${ }^{5}$ W. Rogers, "Elastic property measurement using Rayleigh-Lamb waves," Res. Nondestr. Eval. 6(4), 185-208 (1995).

${ }^{6}$ M. Karim, A. Mal, and Y. Bar-Cohen, "Inversion of leaky Lamb wave data by simplex algorithm," J. Acoust. Soc. Am. 88(1), 482-491 (1990).

${ }^{7}$ J. Deán, C. Trillo, Á. Doval, and J. Fernández, "Determination of thickness and elastic constants of aluminum plates from full-field wavelength measurements of single-mode narrowband Lamb waves," J. Acoust. Soc. Am. 124(3), 1477-1489 (2008).

${ }^{8}$ K. Datta, A. Shah, and W. Subhendu, "Ultrasonic waves and material and defect characterization in composite plates," Mech. Adv. Mater. Struct. 6(4), 285-300 (1999).

${ }^{9} \mathrm{M}$. Castaings and P. Cawley, "The generation, propagation, and detection of Lamb waves in plates using air-coupled ultrasonic transducers," J. Acoust. Soc. Am. 100(5), 3070-3077 (1996).

${ }^{10}$ S. Dahmen, H. Ketata, M. Ghozlen, and B. Hosten, "Elastic constants measurement of anisotropic olivier wood plates using air-coupled transducers generated Lamb wave and ultrasonic bulk wave," Ultrasonics 50(4), 502-507 (2010).

${ }^{11} \mathrm{D}$. Fei, D. Chimenti, and S. Teles, "Material property estimation in thin plates using focused, synthetic-aperture acoustic beams," J. Acoust. Soc. Am. 113(5), 2599-2610 (2003).

${ }^{12}$ M. Kersemans, A. Martens, N. Lammens, K. Van Den Abeele, J. Degrieck, F. Zastavnik, L. Pyl, H. Sol, and W. Van Paepegem, "Identification of the elastic properties of isotropic and orthotropic thinplate materials with the pulsed ultrasonic polar scan," Exp. Mech. 54(6), 1121-1132 (2014).

${ }^{13}$ L. Ambrozinski, B. Piwakowski, T. Stepinski, and T. Uhl, "Evaluation of dispersion characteristics of multimodal guided waves using slant stack transform," NDT\&E Int. 68, 88-97 (2014).

${ }^{14} \mathrm{~W}$. Gao, C. Glorieux, and J. Thoen, "Laser ultrasonic study of Lamb waves: Determination of the thickness and velocities of a thin plate," Int. J. Eng. Sci. 41(2), 219-228 (2003).

${ }^{15}$ C.-H. Yeh and C.-H. Yang, "Characterization of mechanical and geometrical properties of a tube with axial and circumferential guided waves," Ultrasonics 51(4), 472-479 (2011).

${ }^{16}$ L. Yan, H. Cunfu, S. Guorong, W. Bin, C.-H. Chung, and Y.-C. Lee, "Elastic properties inversion of an isotropic plate by hybrid particle swarm-based-simulated annealing optimization technique from leaky Lamb wave measurements using acoustic microscopy," J. Nondestr. Eval. 33(4), 651-662 (2014)

${ }^{17}$ D. Alleyne and P. Cawley, "A two-dimensional Fourier transform method for the measurement of propagating multimode signals," J. Acoust. Soc. Am. 89(3), 1159-1168 (1991).

${ }^{18}$ L. Claes, T. Meyer, F. Bause, J. Rautenberg, and B. Henning, "Determination of the material properties of polymers using laser-generated broadband ultrasound," J. Sens. Sens. Syst. 5(1), 187-196 (2016).

${ }^{19}$ M. Maraschini, F. Ernst, S. Foti, and L. Socco, "A new misfit function for multimodal inversion of surface waves," Geophysics 75(4), G31-G43 (2010).
${ }^{20}$ F. Schöpfer, F. Binder, A. Wöstehoff, T. Schuster, S. von Ende, S. Föll, and R. Lammering, "Accurate determination of dispersion curves of guided waves in plates by applying the matrix pencil method to laser vibrometer measurement data," CEAS Aeronaut. J. 4(1), 61-68 (2013).

${ }^{21}$ J. Fernández, J. Dean, C. Trillo, and Á. Doval, "Elastic constants determination by direct measurement of the beat wavelength between A0 and S0 Lamb modes with pulsed TV holography," Opt. Lasers Eng. 45(5), 618-630 (2007).

${ }^{22}$ K. Xu, D. Ta, D. Cassereau, B. Hu, W. Wang, P. Laugier, and J.-G. Minonzio, "Multichannel processing for dispersion curves extraction of ultrasonic axial-transmission signals: Comparisons and case studies," J. Acoust. Soc. Am. 140(3), 1758-1770 (2016).

${ }^{23}$ B. Hosten, M. Castaings, H. Tretout, and H. Voillaume, "Identification of composite materials elastic moduli from Lamb wave velocities measured with single sided, contactless ultrasonic method," AIP Conf. Proc. 557, 1023-1030 (2001).

${ }^{24}$ J.-G. Minonzio, M. Talmant, and P. Laugier, "Guided wave phase velocity measurement using multi-emitter and multi-receiver arrays in the axial transmission configuration," J. Acoust. Soc. Am. 127(5), 2913-2919 (2010).

${ }^{25}$ N. Bochud, Q. Vallet, J.-G. Minonzio, and P. Laugier, "Predicting bone strength with ultrasonic guided waves," Sci. Rep. 7, 43628 (2017).

${ }^{26}$ A. Leleux, P. Micheau, and M. Castaings, "Long range detection of defects in composite plates using Lamb waves generated and detected by ultrasonic phased array probes," J. Nondestr. Eval. 32(2), 200-214 (2013).

${ }^{27}$ A. Geslain, S. Raetz, M. Hiraiwa, M. Abi Ghanem, S. Wallen, A. Khanolkar, N. Boechler, J. Laurent, C. Prada, A. Duclos, P. Leclaire, and J.-P. Groby, "Spatial Laplace transform for complex wavenumber recovery and its application to the analysis of attenuation in acoustic systems," J. Appl. Phys. 120(13), 135107 (2016).

${ }^{28}$ D. Goldberg, Genetic Algorithms in Search, Optimization and Machine Learning (Addison-Wesley, Reading, MA, 1989).

${ }^{29}$ See supplementary material at https://doi.org/10.1121/1.5024353 for a demonstration of the acquisition process.

${ }^{30}$ A. Briggs and O. Kolosov, Acoustic Microscopy (Oxford University Press, New York, 2010), Vol. 67

${ }^{31}$ C. Prada, D. Clorennec, T. Murray, and D. Royer, "Influence of the anisotropy on zero-group velocity Lamb modes," J. Acoust. Soc. Am. 126(2), $620-625$ (2009).

${ }^{32}$ D. Royer, J.-L. Gennisson, T. Deffieux, and M. Tanter, "On the elasticity of transverse isotropic soft tissues (L)," J. Acoust. Soc. Am. 129(5), 2757-2760 (2011).

${ }^{33} \mathrm{Y}$. Li and R. Thompson, "Influence of anisotropy on the dispersion characteristics of guided ultrasonic plate modes," J. Acoust. Soc. Am. 87(5), 1911-1931 (1990).

${ }^{34}$ S.-H. Rhee, J.-K. Lee, and J.-J. Lee, "The group velocity variation of Lamb wave in fiber reinforced composite plate," Ultrasonics 47(1), 55-63 (2007).

${ }^{35} \mathrm{M}$. Castaings and B. Hosten, "Lamb and SH waves generated and detected by air-coupled ultrasonic transducers in composite material plates," NDT\&E Int. 34(4), 249-258 (2001).

${ }^{36}$ D. Royer, D. Clorennec, and C. Prada, "Lamb mode spectra versus the Poisson ratio in a free isotropic elastic plate," J. Acoust. Soc. Am. 125(6), 3683-3687 (2009).

${ }^{37}$ J. Foiret, J.-G. Minonzio, C. Chappard, M. Talmant, and P. Laugier, "Combined estimation of thickness and velocities using ultrasound guided waves: A pioneering study on in vitro cortical bone samples," IEEE Trans. Ultrason. Ferroelectr. Freq. Control 61(9), 1478-1488 (2014).

${ }^{38}$ F. Honarvar, E. Enjilela, and A. Sinclair, "An alternative method for plotting dispersion curves," Ultrasonics 49(1), 15-18 (2009).

${ }^{39} \mathrm{~F}$. Seco and A. Jiménez, "Modelling the generation and propagation of ultrasonic signals in cylindrical waveguides," in Ultrasonic Waves, edited by A. A. dos Santos (InTech, Madrid, 2012).

${ }^{40} \mathrm{C}$. Prada, O. Balogun, and T. Murray, "Laser-based ultrasonic generation and detection of zero-group velocity Lamb waves in thin plates," Appl. Phys. Lett. 87(19), 194109 (2005).

${ }^{41}$ S. Bernard, G. Marrelec, P. Laugier, and Q. Grimal, "Bayesian normal modes identification and estimation of elastic coefficients in resonant ultrasound spectroscopy," Inverse Probl. 31(6), 065010 (2015).

${ }^{42} \mathrm{P}$. Stoffa and M. Sen, "Nonlinear multiparameter optimization using genetic algorithms: Inversion of plane-wave seismograms," Geophysics 56(11), 1794-1810 (1991).

${ }^{43}$ N. Bochud, Q. Vallet, Y. Bala, H. Follet, J.-G. Minonzio, and P. Laugier, "Genetic algorithms-based inversion of multimode guided waves for cortical bone characterization," Phys. Med. Biol. 61(19), 6953 (2016). 